\title{
Analysis of Dominant and Non-dominant Biceps and Deltoid Muscle Volumes of Badminton Players
}

\author{
Tülin ATAN ${ }^{1}$, Deniz Özge YÜCELOĞLU KESKİN ${ }^{1}$, İlkay ÇAMLIDAĞ ${ }^{2}$, \\ Deniz GÜNAY DEREBAŞI ${ }^{1}$
}

${ }^{1}$ University of Ondokuz Mayıs, Faculty of Yasar Doğu Sports Sciences, Samsun, Turkey

2University of Ondokuz Mayıs, Radiology Department of Medical Faculty, Samsun, Turkey

Address Correspondence Tülin ATAN, takman@omu.edu.tr

\begin{abstract}
The main purpose of this study is to analyze whether there is a difference between dominant and non-dominant biceps and deltoid muscle volumes of badminton players.Also hand grip strengthlevels were compared dominant and non-dominant hands of badminton players.14 registered badminton players between the ages of 11 and 14, who have been playing badminton for at least 5 years and practicing at least 5 days a week, have participated in this study. The muscle volumes of the participants were determined through an MR (1.5 T Philips Achieva Netherlands) device. The T1 weighed MR images of 5 mm section thickness of dominant and non-dominant deltoid and biceps muscle volumes of the participants were obtained, and the lines were drawn at each section at the work station of the related muscle and the sectional area was calculated. The muscle volume was obtained by multiplying the resulting sectional area by the section thickness (Cavalieri principle). Wilcoxon signed rank test was used to analyze the data obtained in this study.It was observed that the dominant deltoid muscle volumes of the players were more than their non-dominant muscle volumes $(\mathrm{p}<0.01)$. Dominant and non-dominant biceps muscle volumes of the players were analyzed and it was observed that the dominant biceps muscle volumes of the players were more than their non-dominant biceps muscle volumes $(\mathrm{p}<0.01)$.Dominant hand grip strength values were found to be higher than nondominant hand $(\mathrm{p}<0.01)$.It was consequently observed that the dominant biceps and deltoid muscle volumes and the dominant grip strength values were higher of badminton players than non-dominant side.These results suggest that the badminton players should also focus on exercises for improving the non-dominant side of their bodies.Otherwise, it can lead to muscle imbalance and injury.
\end{abstract}

Key words: Badminton, Muscle Volume, MR

\section{INTRODUCTION}

The lateralization refers to how the anatomical structure and identical body organs sharing similar characteristics and functions tend to be more dominant and functional in one hemisphere than the other. People tend to prefer their left hand or foot over their right hand or foot or vice versa (19). Lateral dominance is an extremity or a general term for functional dominance of one half of the body. The preferred or better functioning side of the body is clearly observed particularly in athletics (e.g. throwing, bouncing arm, first step, etc.). Highly intense long-term exercises focusing on one side of the body create a risk of morphological asymmetry. One of the paired organs is preferred over the other one (5).

As in other racquet sports, there are short-term maximal or submaximal efforts and short-term resting periods in badminton. Particularly, speed, endurance, strength, coordination, reaction, anticipation, game skills and technique are considered preconditions of success in these sports (4).

When considered in terms of strength and muscular endurance, since the legs, arms and upper part of the body are used in a complicated way when playing badminton, insufficient strength and endurance in upper extremities begin to take effect towards the end of a long rally or the end of the game. The striking power decreases, the player gets distracted and the shape of the body changes (12).

Badminton players favor one particular arm to hold the racket, but it is not known whether this preference causes an asymmetry in the muscles. Too much stress is put on the shoulder of a badminton player. Extraordinary moves are made in order to achieve extra speed. The player creates maximal mobility in order to increase power, and to transform potential energy into kinetic energy to hit the ball. The player pushes his shoulder to its limits 
since hitting the ball with the racquet is a repetitive activity.

The studies show that an exercise performed by one extremity affects the opposite extremity. The results of Tok's (18) research he conducted on healthy male students in Physical Education Teaching Department proved that exhaustion of dominant extremity affects the non-dominant extremity (18).

Therefore, this study was designed to determine whether muscle volume differences between the dominant and non-dominant side is present in the biceps and deltoids of badminton players. Dominant and non-dominant hand grip strengths of badminton players are also compared in this study.

\section{MATERIAL AND METHOD}

Fourteen healthy badminton players who played badminton at least five years (age; 13.07 \pm 2.01 years, height153.64 $\pm 9.18 \mathrm{~cm}$, weight $44.71 \pm 7.28 \mathrm{~cm}$ ) and who exercised at least 6 times per week for 120 min or more participated in the study. Informed consent was obtained from all subjects prior to experimental procedures which were approved by the University Ethical Committee. Each subject filled out a questionnaire on demographics, medical history and physical activity patterns.

\section{Image Analysis}

\section{MRI Protocol}

All MRI examinations were performed on a 1.5 Tesla MRI scanner (Philips, Achieva, Netherlands). A shoulder coil with 8 channel was used for acquisitions. For deltoid muscle imaging, coronal turbo spinecho T1 weighted images were obtained (TR: $18 \mathrm{~ms}$,TE: $556 \mathrm{~ms}$, slice thickness: $3 \mathrm{~mm}$, NSA:2). For bicepsbrachii muscle imaging, axial turbo spinecho T1 weighted images were obtained (TR: 20,TE: 600, slicethickness: 5 mm, NSA:2).

\section{Volumetric image analysis}

All MRI images were transferred to an Osirixim aging software. The borders of the muscle of interest were manually traced on each slice and crosssectional areas were measured in $\mathrm{cm}^{2}$ (Figure 1). Volume of each muscle was calculated according to Cavalieri principle by multiplying the sum of crosssectional areas with the slice thickness. All measurements were performed by a radiologist with 8 years of experience in radiology who were blinded to the knowledge of the side of the dominant arm.
Musclevolume $\left(\mathrm{cm}^{3}\right)=$ Sum of the cross-sectional areas $\mathrm{X}$ slice thickness

Figure 1. Manual delineation of the borders of the deltoid (A) and bicepsbrachii (B) muscles on T1- weighted coronal and axial MR images

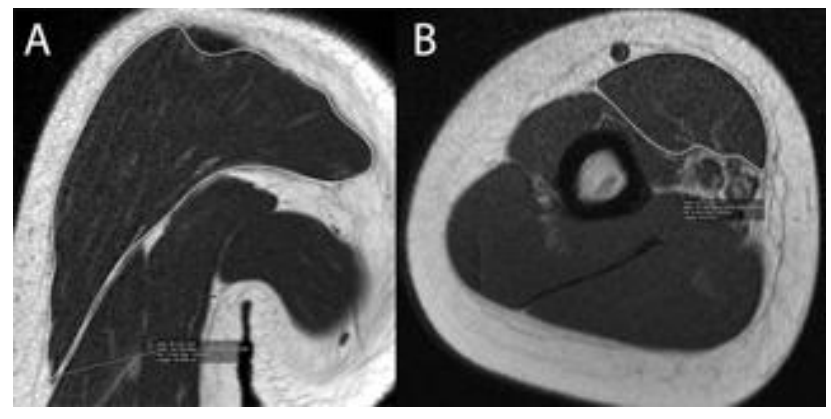

\section{Hand grip strength}

A Takei Physical Fitness Test Grip-D Grip Strength Dynamometer, which measures strength between 0 and $100 \mathrm{~kg}$, was used to measure hand grip strength. Dynamometer was adjusted for the hand size of each participant. After warming up for five minutes, the measurements were taken when the participants were in standing position, without bending their arms, and their arms were at a $15^{\circ}$ angle and not touching their own bodies. This process was repeated three times for each hand and the highest value was used.

\section{Heights of the participants}

The heights of the participants were measured with a cm-scale measurement scale.

\section{Body weight measurements}

The participants were barefoot and wearing shorts and their body weights were measured on a body weight scale.

\section{Statistical Analysis}

As a result of the power analysis performed to determine the number of participants, it was decided that 14 participants would be sufficient to achieve $85 \%$ of test power at 2 units of mean difference and 4 units of standard deviation. Wilcoxon signed rank test were used to compare the data obtained in this study. Statistical significance was determined as $\mathrm{p}<0.05$. SPSS version 21.0 was used to perform statistical analysis of the data obtained in this study. 


\section{RESULT}

Table 1. Dominantand non-dominant biceps and deltoid muscle volumes of badminton players

\begin{tabular}{|c|c|c|c|c|c|c|c|}
\hline \multicolumn{2}{|c|}{ Muscle volume } & \multirow{2}{*}{$\begin{array}{l}\text { Mean } \\
1749,85\end{array}$} & \multirow{2}{*}{$\begin{array}{l}\text { Standard } \\
\text { Deviation }\end{array}$} & \multirow{2}{*}{$\begin{array}{c}\text { Median } \\
1370.50\end{array}$} & \multirow{2}{*}{$\begin{array}{l}\text { Min } \\
718,00\end{array}$} & \multirow{2}{*}{$\begin{array}{l}\text { Max } \\
2922,00\end{array}$} & \multirow{2}{*}{$\begin{array}{l}\mathrm{p} \\
0.001^{* *}\end{array}$} \\
\hline Deltoid & Dominant & & & & & & \\
\hline$\left(\mathrm{cm}^{3}\right)$ & Non-Dominant & 1317,71 & 595,51 & 1099.00 & 681,00 & 2541,00 & \\
\hline Biceps & Dominant & 657,64 & 276,45 & 571.00 & 360,00 & 1192,00 & \multirow{2}{*}{$0.001^{* *}$} \\
\hline$\left(\mathrm{cm}^{3}\right)$ & Non-Dominant & 565,64 & 294,30 & 484.00 & 207,00 & 1133,00 & \\
\hline
\end{tabular}

$$
{ }^{* *} \mathrm{p}<0.01
$$

A statistically significant difference was observed between the dominant and non-dominant deltoid muscle volumes of the players. It was observed that the dominant deltoid muscle volumes of the players were more than their non-dominant muscle volumes $(\mathrm{p}<0.01)$.Dominant and non-

dominant biceps muscle volumes of the players were analyzed and a statistically significant difference was observed between these muscle volumes. It was observed that the dominant biceps muscle volumes of the players were more than their non-dominant biceps muscle volumes $(p<0.01)$.

\begin{tabular}{llllllll}
\hline \multicolumn{7}{l}{ Table 2. Dominant and non-dominant hand grip values of badminton players } \\
\hline & Mean & $\begin{array}{l}\text { Standard } \\
\text { Deviation }\end{array}$ & Median & Min & Max & $\mathrm{p}$ \\
\hline \multirow{2}{*}{$\begin{array}{l}\text { Hand grip } \\
\text { strength }(\mathrm{kg})\end{array}$} & Dominant & 20,77 & 6,05 & 20.55 & 13.40 & 31.00 & \\
\cline { 2 - 6 } & $\begin{array}{l}\text { Non- } \\
\text { Dominant }\end{array}$ & 18,30 & 5,65 & 18.95 & 10.50 & 28.00 & \\
\hline
\end{tabular}

${ }^{* *} \mathrm{p}<0.01$

Dominant and non-dominant hand grip strength values of the badminton players were compared and the grip strength of their dominant hands were found to be higher than the other hand $(\mathrm{p}<0.01)$.

\section{DISCUSSION}

In this study, it was aimed to find out whether there were any differences between the dominant biceps and deltoid muscle volumes and nondominant biceps and deltoid muscle volumes of badminton players. It was observed that the dominant deltoid muscle volumes of the players were more than their non-dominant muscle volumes. Again, dominant and non-dominant biceps muscle volumes of the players were analyzed and it was observed that the dominant biceps muscle volumes of the players were more than their non-dominant muscle volumes. The grip strength of their dominant hands was also found to be higher than the other hand. It was reported that the dominant hand is an effective factor on hand grip strength (1). The reason of this result could be due to the nature of badminton in which players repeatedly hold the racket and hit the ball with a dominant arm.

Similar studies, which reported the difference between the dominant and non-dominant sides of the players, were also found in literature $(6,7,9)$.

Ducher et al. (6) investigated the effects of longterm tennis playing on the relationship between lean tissue mass and bone mineral content in the forearms, taking the body dimensions into account. Fifty-two tennis players were recruited. They measured lean tissue mass, bone area, bone mineral content (BMC), and bone mineral density at the forearms from a DXA whole-body scan. They assessed grip strength with a dynamometer. They found a marked side-to-side difference in favor of the dominant forearm in all parameters. They assessed bone area and BMC correlated with grip strength on both sides. They found the correlations were still significant after adjusting for whole-body BMC, body height, or forearm length. Their study result reinforced that, the putative role of the muscles in the mechanical loading on bones (6). 
Pennock et al. (13) examined abnormalities noted on magnetic resonance imaging (MRI) in the shoulders of asymptomatic Little League baseball players. The dominant arm was 8.5 times more likely to have an abnormality on MRI compared with the nondominant arm. In all, 12 players (52\%) had 17 positive MRI findings in their throwing shoulder that were not present in their nondominant shoulder (13).

Kong and Burns (9) compared bilaterall strength characteristics of the hamstrings and the quadriceps muscle groups in recreationally active, healthy males and females. Their main findingwas $\mathrm{H}$ :Q ratio was higher in the dominant than the nondominant legs for both isometric and isokinetic measurements (9).

A study by Ellenbecker (7) conducted on 22 professional male tennis players showed that all players had high levels of isokinetic strength, and the average internal rotation peak torque value was higher in the dominant side than the non-dominant side. Significant differences were found between the dominant and non-dominant shoulders in shoulder rotation moves (7).

Grip strength is considered an objective measurement in upper extremity performance evaluation (11). Peterson et al. (15) found that the grip strength of the dominant hand is $10 \%$ more than the grip strength of the non-dominant hand (15). In another study dominant hand grip strength were found to be higher and statistically significant compared to non-dominant hand grip strength (10).

In another study on soccer players where there was a greater than $10 \%$ difference in muscle strength between the knee flexors of the dominant legand the non-dominant leg, a total of 28 of the 41 players $(68 \%)$ had significant musculoskeletal abnormality (imbalance $>10 \%$ ) in one or more specific muscle groups (16).

Another study reported that the dominant shoulders of tennis players have significantly more scapular and glenohumeral mobility than their nondominant shoulders. Poor body mechanics, muscular exhaustion or weakness that emerges following these extreme moves may result in injuries. This risk depends on the balance between the mobility and the stability of the shoulder (2).

Although our data is in alignment with the study by Ducher et al. (6) and Kong and Burns (9), it is contradictory to a published study by Rosene et al. (17). They have found no difference between the dominant and non-dominant legs in collegiate athletes.

Turk ل Sport Exe 2019; 21(2): 323-327

(4) 2019 Faculty of Sport Sciences, Selcuk University
It is not clear what causes this discrepancy between the studies. It might be a sports branch issue since our study was conducted on badminton players where as volleyball, soccer, basketball and softball players were involved in the study by Rosene et al. (17). Badminton players use dominant arm too much so this could be the reason.

Based on the previous studies, which showed no differences between the dominant and nondominant sides of non-athletes (3), it may be concluded that the difference observed in athletes is related to their training activity. In other words, training may be more relevant for the reasons of the difference between dominant and non-dominant sides.

Armstrong and Oldham's study (3) compared dominant and non-dominant hand strength in both right- and left-handed participants in non-athletes. Their study group was recruited from hospital staff and visitors and the staff and students of a local university college. Maximum voluntary contraction of the first dorsal interosseous muscle, power grip strength and pulp-to-pulp pinch strength were assessed under carefully controlled conditions. They observed no significant differences between dominant and non-dominant hands in left-handed participants for all tests. They observed small but significant differences $(0.1-3 \%)$ between dominant and non-dominant hands in right-handed participants for all three tests (3).

Extreme and repetitive moves put the static stabilizers (glenohumeral ligaments, labrum, and bone structure) of the shoulder under stress. Extreme moves during the game and tightening of these stabilizers increase the player's performance. However, this may cause underperformance, shoulder instabilities or injuries in the long run. In order to prevent these problems, each player should be well-trained on maintaining the balance between shoulder mobility and stability. This training process should start early in the career of the player $(8,14)$.

It was consequently observed that the dominant biceps and deltoid muscle volumes of badminton players were higher. It was also observed that the grip strength of badminton players' dominant hands was also found to be higher than the other hand. 


\section{CONCLUSION}

Therefore, while planning the training programs of badminton players, considering

\section{REFERENCES}

1. Akarsu, S. Relations among handedness, reaction time, menocular shifts from binocular focal point and IQ in sedentary subjects and athletes in different sports. Master thesis. University of Atatürk. Physiology Department. Erzurum,2004.

2. Akça, G. Evaluation of gleno humeral joint range of motion, scapular dyskinesis and shoulder proprioception in elite tenis players. Master thesis.Institute of Health Sciences.University of 9 Eylül. İzmir, 2006.

3. Armstrong, C. A., \& Oldham, J. A. A comparison of dominant and non-dominant hand strengths. Journal of Hand Surgery (British and European Volume), 1999; 24(4): 421-425.

4. Baron, R., Petschnig, R., Bachl, N., Raberger, G., Smekal, G., \& Kastner, P. Catecholamine excretion and heart rate as factors of psychophysical stress in table tennis. Int J Sports Med, 1992; 13(7): 501-5.

5. Beyer, E., \& Hofmann, V. K. Dictionary of Sport Science. Schorndorf, 1992.

6. Ducher, G., Jaffré, C., Arlettaz, A., Benhamou, C. L, \& Courteix, D. Effects of long-term tenis playing on the musclebone relationship in the dominant and non-dominant forearms. Canadian Society for Exercise Physiology. Can. J. Appl. Physiol, 2005; 30(1): 3-17.

7. Ellenbecker, T. S. A total arm strength isokinetic profile of highly skilled tenis players. Isokinetics and Exercise Science,1991; 1(1): 9-21.

8. Griffin, K. M., Bonci, C., \& Sloane, B. Prevention and rehabilitation of shoulder injuries in throwing athletes. In: NicholasJA, Hershman EB, PosnerMA, editors. The Upper Extremity In Sports Medicine. 2nd ed. USA :Churcill Livingstone pres, 1995: 721-747.

9. Kong, P. W., \& Burns, S. F. Bilateral difference in hamstrings to quadriceps ratio in healthy males and females. Physical Therapy in Sport, 2010;11(1): 12-17.

10. Mitsionis, G., Pakos, E.E., Stafilas, K.S., Paschos, N., Papakostas, T., \& Beris, A.E. Normative data on hand grip strength in a Greek adult population. International Orthopaedics,2009; 33(3): 713-717.

11. Ng, G. Y., \& Fan, A. C. Does elbow position affect strength and reproducibility of power grip measurements? Physiotherapy,2001; 87(2): 68-72.

12. Omesegaard, B. O. IBF Physical Training For Badminton. Mailingbeck A/S. Denmark, 1996: p37-51.

13. Pennock, A. T., Dwek, J., Levy, E., Stearns, P., Manning, J., Dennis, M. M., Davis-Juarez, A., Bastrom, T., Taylor, K. S. Shoulder MRI abnormalities in asymptomatic little league baseball players. Orthopaedic Journal of Sports Medicine,2018; 6(2): 1-6 individual characteristics depending on laterality may both increase performance and decrease the risk of injuries.

14. Perry, J., \& Glausman, R. E. Biomechanics of throwing. In: Nicholas JA, Hershman EB, Posner MA, editors. The Upper Extremity In Sports Medicine. 2nd ed. USA :Churcill Livingstone pres,1995:697-719.

15. Peterson, P., Petrick, M., Connor, H., \& Conklin, D. Grip strength and hand dominance: challenging the $10 \%$ rule. Am J OccupTher, 1989;43(7): 444-447.

16. Rahnama, N., Lees, A., \& Bambaecichi, E. A comparison of muscle strength and flexibility between the preferred and non-preferred leg in English soccer players. Ergonomics,2005; 48(11-14): 1568-1575.

17. Rosene, J. M, Fogarty, T. D., \& Mahaffey, B. L. Isokinetic hamstrings: quadriceps ratios in inter collegiate athletes. Journal of Athletic Training,2001; 36(4): 378-383.

18. Tok, M. I. The effects of vibration training on spinal excitability. PhD thesis, University of Ege, Institute of Health Sciences. İzmir, 2007.

19. Yazıc1, A. G. Comparison between lateralization levels of athletes who do exercises actively and their dynamic and static balance and some physical features. $\mathrm{PhD}$ thesis. Institute of Health Sciences.University of Atatürk. Erzurum, 2012.

Notes: Note 1 . This study has been supported by Ondokuz MayısUniversity with the reference number PYO.YDS.1901.15.001 